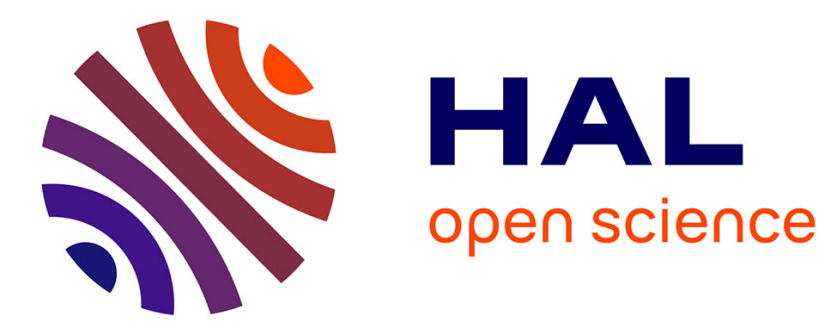

\title{
Damping analysis of a free aluminum plate
}

Lionel Zoghaib, Pierre-Olivier Mattei

\section{To cite this version:}

Lionel Zoghaib, Pierre-Olivier Mattei. Damping analysis of a free aluminum plate. Journal of Vibration and Control, 2013, pp.1077546313507098. 10.1177/1077546313507098 . hal-01024479

\section{HAL Id: hal-01024479 \\ https://hal.science/hal-01024479}

Submitted on 17 Jul 2014

HAL is a multi-disciplinary open access archive for the deposit and dissemination of scientific research documents, whether they are published or not. The documents may come from teaching and research institutions in France or abroad, or from public or private research centers.
L'archive ouverte pluridisciplinaire HAL, est destinée au dépôt et à la diffusion de documents scientifiques de niveau recherche, publiés ou non, émanant des établissements d'enseignement et de recherche français ou étrangers, des laboratoires publics ou privés. 
1 Damping analysis of a free aluminum plate

\author{
Lionel Zoghaib \\ EADS, France \\ Pierre-Olivier Mattei ${ }^{1}$ \\ (LMA,) CNRS ; Aix-Marseille Univ ; Centrale Marseille, France
}

\begin{abstract}
An analysis of the energy dissipation sources acting in a vibrating aluminum plate is presented in this paper. In a first step, the contact-free modal analysis of a suspended plate is conducted using a laser vibrometer and an acoustic excitation to obtain reference data. The thin nylon suspension set-up guarantees a low boundary damping, which is assumed to be negligible. In a second step, a number of damping sources are modeled. Acoustic damping due to the noise radiation of the non-baffled plate is computed using the boundary integral method and a light fluid approximation to express the vibroacoustic coupling in analytical terms. The damping due to the sheared air flow along the free plate borders is determined on the basis of a simple two-dimensional boundary layer model. Thermoelastic damping is assessed using a Fourier series expression for the temperature field along with a perturbation technique to take thermoelastic coupling into account. Since no robust model is available so far to quantify viscoelastic material damping in aluminum, it is determined in a last step by subtracting measured values of damping to the one that have previously been computed. Aluminum viscoelastic damping turns out to be very small and almost independent of frequency.
\end{abstract}

\title{
Keyword
}

Damping, thermoelastic damping, viscoelastic damping, material damping, acoustic radiation damping

\section{Introduction}

While a broad literature exists on the subject of damping, a few authors only have performed a systematic analysis of all dissipation sources in structures. Among them, Cuesta and Valette (1993) have made an interesting contribution on strings damping by quantifying several dissipation sources such as thermoelastic damping, viscoelastic material damping and air flow damping. Lambourg and Chaigne (2001) have also published a theoretical and experimental study on the damping in wood and metal plates. The authors have modeled and identified a number of dissipation sources for the application field of sound synthesis. In the present paper, which focuses on the processes that most contribute to damping in an aluminum plate, the strategy to model rather than to identify them has been chosen whenever possible. The aim, indeed, is to give a better insight into the physics of dissipation. The drawback of this strategy, however, is that a complete quantification of the main dissipative phenomena is required to be able to validate the model predictions, since the only accessible quantity experimentally is the overall damping. A systematic evaluation of all damping components involves a number of disciplines such as tribology, acoustics or thermomechanics, which make a detailed analysis particularly challenging.

45 A first step is to draw up an inventory of all dissipation sources. This has already been 46 carried out some time ago by Zener (1948) as far as aluminum plates concerns. According 47 to him, thermoelastic damping is the main damping component in aluminum due to the

\footnotetext{
${ }^{1}$ Corresponding author. LMA, CNRS, UPR 7051, Aix-Marseille Univ, Centrale Marseille, F-13402 Marseille Cedex 20, France
} 
material high conductivity and compressibility. Cremer et al. (1988) have also pointed out the existence of a damping component related to microstructural viscoelasticity. Its value is generally assumed to be rather small and constant in most studies. From a structural perspective, the boundary area where the plate is being fixed can heavily contribute to damping. Physical phenomena involved in this area such as friction, thermoelasticity or energy leakage are particularly complex and have a great influence on the overall structural behavior. The first two of them require a local description of the physics at the interface, whereas energy leakage can only be handled properly by modeling the neighborhood and trimming it where the energy transmission is found negligible, for instance where the vibration energy starts being very low. In the present paper, a drastic simplification of the boundary modeling has been aimed at by suspending the plate with two thin nylon wires. These wires are poor thermal conductors and are also assumed to be not very dissipative. They can be idealized by a string model, for which the transverse stiffness is determined by the weight of the plate, and consequently apply a very low restoring force to the plate boundary. Boundary dissipation is thus taken to be negligible and the structural configuration considered as free.

Apart from material damping, two additional boundary damping components involving the air surrounding the structure have also been investigated here. The first one, called acoustic damping, accounts for the vibrational energy being lost due to the noise radiation in the air. It requires an extension of the domain to take the exterior fluid into account. Boundary conditions such as Sommerfeld far field condition are also needed to trim the fluid domain. The second damping is due to the air flow along the structure edges. Large bending displacements take place at the edges of the suspended plate, since it is almost free. The fluid is sheared upon by flowing tangentially to the plate thickness and thus causes energy dissipation to occur.

The paper is constructed as following. First, data from an experimental modal analysis of an aluminum plate is gathered and presented. The measured modal damping is considered as a reference value of the total damping since it results from the contribution of a variety of dissipation sources. The rest of the paper is then aiming at understanding what this total damping is essentially made of. The fluid-structure interactions are first evaluated ; air flow damping as well as acoustic radiation damping, considered as the most important sources, are modeled and computed. Two main sources of material damping are then considered in a final part: thermoelastic and viscoelastic damping. While thermoelastic damping can still be modeled and assessed, no satisfactory model is currently available to estimate the viscoelastic damping precisely. This final dissipation source, due to friction micromechanisms, has thus to be identified. It is deduced in a last step from all modeled and measured values.

\section{Measurement of the overall damping of a suspended plate}

The modal analysis of a $35 \mathrm{~cm} \times 40 \mathrm{~cm} \times 2 \mathrm{~mm}( \pm 0.1 \mathrm{~mm})$ aluminum plate, weighting $190.5 \mathrm{~g}$, has been carried out to obtain a reference frequency and an overall value of damping for each mode. To be able to suspend the plate, two tiny bores have been drilled out on one edge; a thin nylon wire has then been embedded and bonded at each bore. A special care has been taken to reduce as much as possible any source of joint damping at the attachment. Since rigid modes can easily be excited in configurations of this kind, a contact-free set-up has been implemented with a laser vibrometer (polytech OFV 303) to measure the plate velocity and a loudspeaker to excite it (figure 1). Signals have been generated and processed by a Hewlett Packard acquisition system (paragon HP356xa). A high-pass filter 
and an amplifier have been set between the signal generator and the loudspeaker in order to suitably tune the excitation signal and to remove damaging low frequency components.

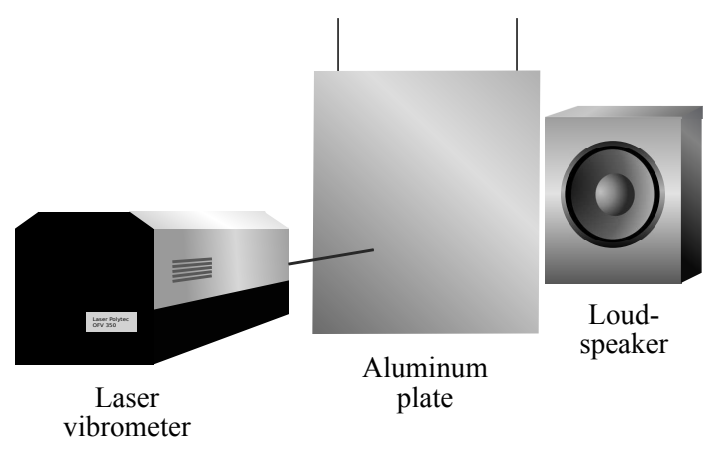

Figure 1: Contact-free modal analysis set-up

The classical decay method, particularly appropriate to make accurate measurements of damping in frequency dependent systems, has been used (Rao, 2010; Nashif et al., 1985). The method consists of two main steps. In a first step, a broadband excitation is generated to detect the plate resonance frequencies, which are the free solutions of the vibroacoustic system. A very good approximation of these frequencies can be estimated in the low frequency range where peaks appear on the spectrum, at least for low damping cases. For larger modal overlap configurations, the contribution of other modes can pollute the frequency identification by slightly shifting the peak location. The effect is considered to be negligible here due to the very low damping values and the wide gap between modal frequencies. In a second step, the damping of each low frequency resonance mode is studied one after the other by emitting a pure sine wave. The frequency of the sine is tuned to that of the considered resonance mode. After a short pause, the excitation signal is switched off and the velocity decay observed. Using a logarithmic scale, the signal envelope is found to take the form of a straight line, the slope of which is a measure of damping. No pollution from close modes has been observed and only clean unequivocal measurements have been reported. The procedure has been repeated for several measurement points without any noticeable change. The relation between the measured damping $\alpha$ (the straight line slope) and the complex resonance angular frequency $\Omega$, which can be computed numerically using an eigenvalue solver, is simply given by $\Omega=\omega+i \alpha$, where $\omega$ is the real angular frequency in rad.s $\mathrm{s}^{-1}$ and $\alpha$ the imaginary part of the angular frequency expressed in $\mathrm{Hz}$. This damping definition, although quite unusual, will be used throughout the article since it has a strong numerical and experimental meaning. It is also a direct measure of the amplitude weighting applied by dissipation at the resonance. Classical measures of damping, such as the loss factor $\eta$ or the inverse of the quality factor $Q^{-1}$ can easily be deduced using the formula $\eta=Q^{-1}=\alpha / \pi f$. The shape, frequency and damping of 26 out-of-plane modes ranging from $43.4 \mathrm{~Hz}$ to $839 \mathrm{~Hz}$ have been identified. Their main characteristics are summarized in table 1. This frequency domain will be considered throughout the article and sets validity bounds for the analysis presented here. Frequencies have been measured with a $0.125 \mathrm{~Hz}$ resolution, while shapes have been obtained by scanning the whole plate with two meshes of either $16 \times 16$ or $32 \times 32$ points, depending on the shape pattern complexity. Damping measurements are displayed in figure 2. It can be noticed that damping varies quite significantly from a mode to another. This modal behavior, due to the fact that some areas damp more than others within the structure, will be identified later as resulting from the plate thermoelasticity. A consequence of damping non-proportionality is the existence of complex modes. A complex eigensolver or any alternative technique (Adhikari, 2011; Cha, 2005; Cortés and Elejabarrieta, 2006) such as the perturbation method used in this article 


\begin{tabular}{|c|c|c|c|c|c|c|c|}
\hline Mode & $(1,1)$ & $(0,2)$ & $(2,0)$ & $(1,2)$ & $(2,1)$ & $(\mathbf{0 , 3})$ & $(2,2)$ \\
\hline Frequency $[\mathrm{Hz}]$ & 43.40 & 62.25 & 93.75 & 111.75 & 124.38 & 191.13 & 218.38 \\
\hline Damping $[\mathrm{Hz}]$ & $\overline{0.13}$ & 0.37 & 1.11 & 0.49 & $\overline{0.51}$ & 1.17 & 0.74 \\
\hline Mode & $\overline{(1,3)}$ & $\overline{(3,0)}$ & (3,1) & $\overline{(2,3)}$ & $\overline{(0,4)}$ & $\overline{(3,2)}$ & $\overline{(1,4)}$ \\
\hline Frequency $[\mathrm{Hz}]$ & 226.13 & 248.13 & 288.00 & 352.25 & 372.00 & 376.50 & 419.50 \\
\hline Damping $[\mathrm{Hz}]$ & 0.73 & 1.28 & 1.29 & 0.93 & 1.32 & 1.01 & 1.33 \\
\hline Mode & $\overline{(4,0)}$ & $\overline{(\overline{(4,1)}}$ & (3,3) & (2,4) & (0,5) & $\bar{~} \overline{(4,2)}$ & $\bar{~} \overline{(1,5)}$ \\
\hline Frequency $[\mathrm{Hz}]$ & 487.25 & 518.88 & 530.75 & 538.75 & 619.13 & 624.25 & 654.38 \\
\hline Damping $[\mathrm{Hz}]$ & 1.71 & 1.50 & 1.19 & 1.30 & 1.74 & 1.62 & 1.61 \\
\hline Mode & $\overline{(3,4)}$ & $\overline{(\overline{(4,3)}}$ & $\overline{(2,5)}$ & 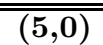 & $\overline{(\overline{(5,1)}}$ & & \\
\hline Frequency $[\mathrm{Hz}]$ & 727.00 & 765.13 & 795.63 & 809.50 & 839.00 & & \\
\hline Damping $[\mathrm{Hz}]$ & 1.41 & 1.43 & 1.67 & 2.11 & 1.99 & & \\
\hline
\end{tabular}

Table 1: Identified modes of the $35 \mathrm{~cm} \times 40 \mathrm{~cm} \times 2 \mathrm{~mm}$ free aluminum plate

needs thus to be implemented to properly account for the structure dynamic behavior.

\section{Fluid-structure interaction damping}

\subsection{Airflow damping}

\subsubsection{Introduction}

The viscosity of air causes energy to be dissipated via compression and shear. While compression dissipation is assumed to be negligible here, shear dissipation could possibly be important in the vicinity of the plate free edges. In this area, indeed, air flows tangentially to the border. Air viscosity can play a significant role in a number of situations, for instance in the case of wave propagation in ducts. It has also been identified as a major source of dissipation in strings in the low frequency range by Cuesta and Valette (1993). The authors have used Stokes formalism to model the laminar flow around a cylinder with a low Reynolds number hypothesis. Landau and Lifschitz' equations (Landau and Lifschitz, 1986) have been used here instead, which provide with a viscous friction caused by the motion of a flat infinite surface in its plane. The extension to the finite surface case is considered by including a correction term. As opposed to Blasius solution (Cousteix, 1988), which describes the boundary layer created by a two-dimensional incompressible flow on a halfinfinite plane, non-linear terms are neglected but inertial terms kept. Blasius solution gives a variable boundary layer thickness, whereas the present model gives a boundary layer with a constant thickness. Based on the hypothesis of stationary motion, it results in a linear viscous damping mechanism.

\subsubsection{Two-dimensional analytical flow modeling}

The method description is illustrated by the simplified geometry shown in figure 3 .

The plate edge is associated with an infinite line $L$ that corresponds to an infinitely thick plate. The edge has an up-right stationary motion along $L$ of amplitude $u(s)$ in Laplace domain. Laplace parameter $s$ is linked to the angular frequency $\omega$ via $s=i \omega$. Pressure $p(x, y, z, s)$ and velocity $v_{i}(x, y, z, s)(i \in\{x, y, z\})$ of an air particle are the unknowns of the problem. By assuming that the system is invariable with respect to any translation along $z$, that the motion in the fluid is driven by the fluid-structure coupling only, and incompressibility, the components of Navier-Stokes equation become:

$$
\left\{\begin{aligned}
p_{, x}(x, s) & =0 \\
s v_{z}(x, s) & =\nu v_{z, x x}(x, s)
\end{aligned}\right.
$$




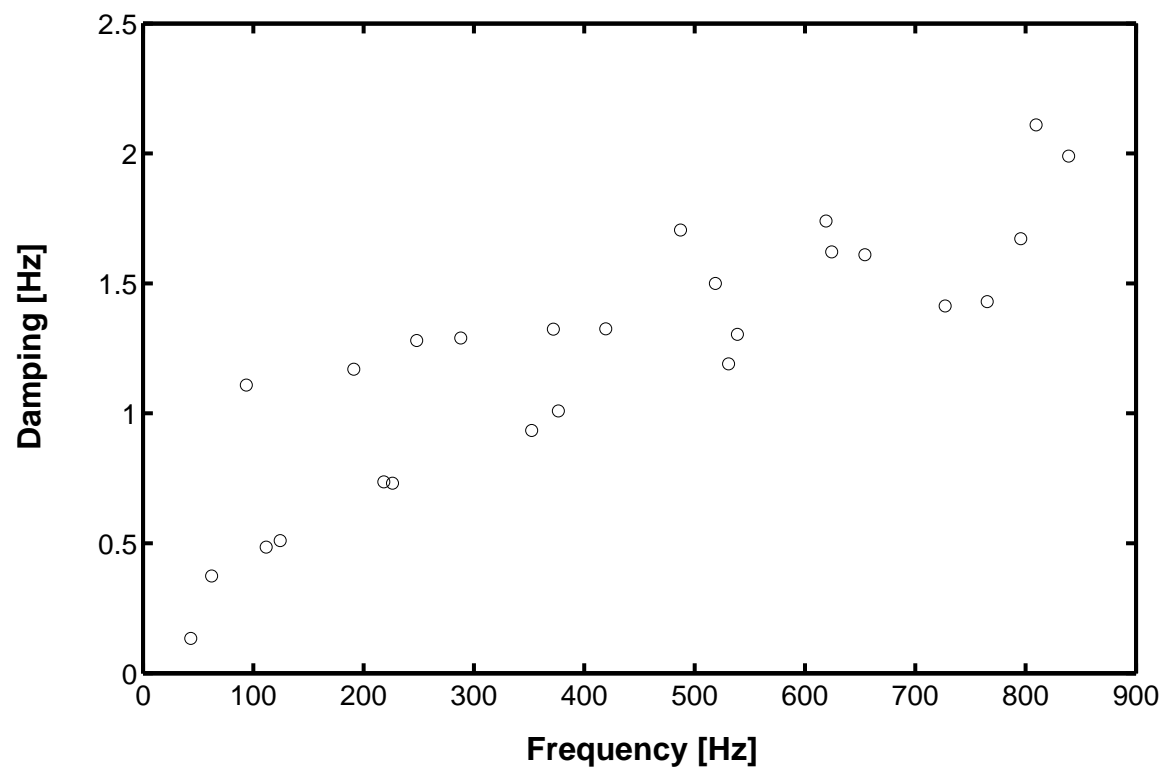

Figure 2: Aluminum plate measured damping

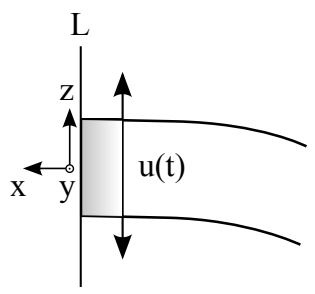

Figure 3: 2-D flow model along the edge of a free plate experiencing bending motion 
where $\nu$ is the kinematic viscosity. All details of the calculation can be found in Landau and Lifschitz' textbook (Landau and Lifschitz, 1986). The first equation shows that pressure is constant. The second one is a diffusion equation. If $v_{z}(x, s)$ is given the form $v_{z}(x, s)=$ $e^{i k x} u(s)$ and inserted in equation (1), following result is obtained:

$$
\left\{\begin{aligned}
k & = \pm i \sqrt{\frac{s}{\nu}} \\
v_{z}(x, s) & =e^{\sqrt{\frac{s}{\nu}} x} u(s)=e^{\sqrt{\frac{\omega}{2 \nu}}(1+i) x} u(s)
\end{aligned}\right.
$$

The complex plane offers two solutions. The only physical one is associated to a highly damped shear wave that propagates in the fluid. The wave penetration depth $\delta=\sqrt{\frac{2 \nu}{\omega}}$ can be compared to Cuesta and Valette's result $\delta=\sqrt{\frac{\nu}{2 \omega}}$ obtained in the case of a flow along a cylinder. These expressions for $\delta$ are consistent in terms of kinematic viscosity and frequency dependence. Blasius solution, which assumes a incompressible non-linear flow with no inertia, gives a boundary layer thickness $\delta=5 \sqrt{\frac{\nu z}{u}}$ that depends also on $\mathrm{z}$ and the flow constant velocity $u$. An illustration of the boundary layer thicknesses obtained with Blasius solution and the present model, based on Landau and Lifschitz equations, is displayed in figure 4 . The following tangential shear stress results in the fluid of density $\rho_{f}$ :
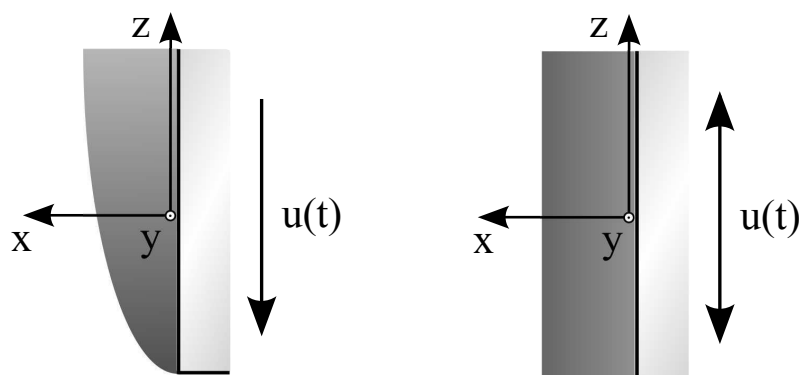

Figure 4: Boundary layer obtained with Blasius solution (half-plane, non-stationary flow) and with Landau and Lifschitz's approach (infinite plane, stationary flow)

$$
\sigma_{z x}^{F}(x, s)=\rho_{f} \nu v_{z, x}(x, s)=\rho_{f} \sqrt{\nu s} e^{\sqrt{\frac{s}{\nu} x}} u(s)
$$

As a reaction, a transverse shear stress is generated within the solid along the interface $L$ :

$$
\sigma_{z x}^{S}(z, s)=\sigma_{z x}^{S}(s)=\rho_{f} \sqrt{\nu s} u(s) \quad(\text { over } L)
$$

In the case of a half-plane oscillation, a border correction term equivalent to an increase of $\delta / 2$ in the area swept by the half-plane is added by Landau and Lifschitz. Cuesta and Valette (1993) have also made a correction of Stokes solution after noting that the boundary layer thickness $\delta$ is similar to the cylinder radius.

\subsubsection{Numerical results}

This analytical solution can easily be introduced into a modal analysis program based on the finite element method and a standard eigenvalue solver, which computes the eigenpairs $(\lambda, u)$ of the system formed by the stiffness matrix $K$ and the mass matrix $M$ of the structure. A flow stress matrix $K^{\text {flow }}$, built up according to equation (4), can be introduced in a new eigenvalue problem associated to new eigenpairs $(\Lambda, U)$ :

$$
\left(K+K^{\text {flow }}-\Lambda M\right) U=0
$$


Assuming that $K^{\text {flow }}$ has only a slight influence on the overall system, it can be regarded as a perturbation matrix (Woodhouse, 1988). The eigenvectors of the system in vacuum are used as a projection basis without the need for a complex solver and a specific strategy to handle frequency dependence. The new resonance values are deduced from those of the unperturbed system by using following expression:

$$
\Lambda \sim \frac{u^{T}\left(K+K^{\text {Flow }}\right) u}{u^{T} M u}=\lambda+u^{T} K^{\text {flow }} u
$$

where vectors have first been mass-orthonormalized. The method's precision is not known $a$ priori but it can be very high if the perturbation is small, as confirmed by results a posteriori. The computation has been carried out with a finite element program that implements a 27nodes solid element and a Gauss full integration scheme. Convergence checks have been performed with various mesh densities and have shown that the selected element size is satisfactory for all considered modes. A summary of the model physical characteristics is displayed in table 2. Numerical air flow damping values expressed in $\mathrm{Hz}$ are given in

\begin{tabular}{|l|l|}
\hline Dimensions & $35 \mathrm{~cm} \times 40 \mathrm{~cm} \times 2 \mathrm{~mm}$ \\
Young's modulus & $70 \mathrm{GPa}$ \\
Poisson ratio & 0.3 \\
aluminum density & $2700 \mathrm{~kg} \cdot \mathrm{m}^{-3}$ \\
Air density & $1.3 \mathrm{~kg} \cdot \mathrm{m}^{-3}$ \\
Sound velocity & $340 \mathrm{~m} \cdot \mathrm{s}^{-1}$ \\
Kinematic viscosity & $1.38510^{-5} \mathrm{~m}^{2} . \mathrm{s}^{-1}$ \\
\hline
\end{tabular}

Table 2: Model characteristics

figure 5. These values have been found to be several orders lower than other damping sources. Although air flow damping -when expressed as a loss factor- is stronger in the low frequency range, in line with Cuesta and Valette's findings for the case of strings, it can be said to be negligible in the current configuration.

\subsection{Acoustic radiation damping}

\subsubsection{Introduction}

Bending motion can induce dissipation by transmitting vibrational energy to the surrounding air via noise radiation. To model this phenomenon, the finite element program described previously has been used to model the dynamics of the plate in vacuum in combination with a boundary element program. Based on the indirect integral formulation and applied to a thin finite screen in an infinite fluid medium, it is able to compute the dipole part of the acoustic radiation. Both routines have been linked via a perturbation technique that accounts for the vibroacoustic coupling and that eventually determines an acoustic radiation damping.

\subsubsection{Radiation of a non-baffled plate}

Many authors, including Laulagnet (1998), Côté et al. (1998) and Atalla et al. (1996) have studied the radiation of non-baffled plates, using Helmholtz equation and the associated Green integral formulation of the acoustic pressure. A similar computation, adapted to the current situation that aims at assessing damping, is presented now. Assuming that the plate 


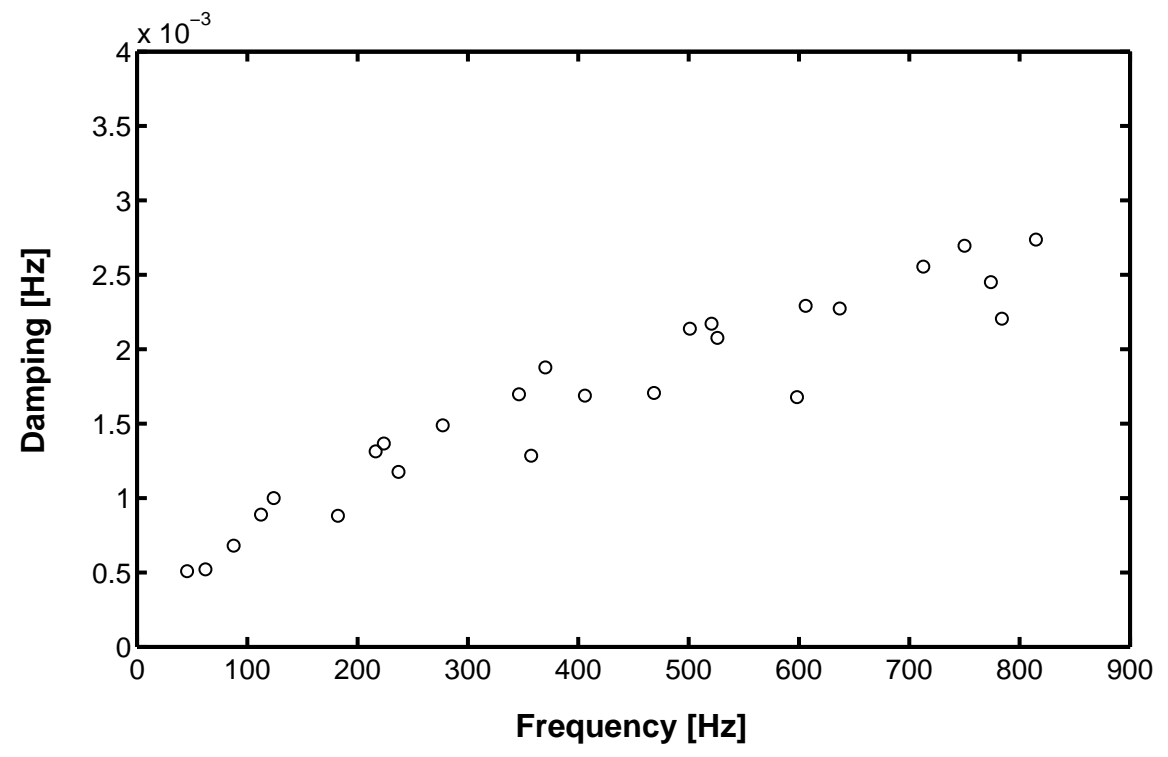

Figure 5: Airflow damping of a free $35 \mathrm{~cm} \times 40 \mathrm{~cm} \times 2 \mathrm{~mm}$ aluminum plate

is thin and oriented perpendicularly to the direction $z$, an indirect integral formulation of the problem can be written as following (Filippi et al., 1999):

$$
\begin{aligned}
p\left(M_{0}, s\right) & =\rho_{f} s^{2} \int_{S}\left(u_{+}(M, s)-u_{-}(M, s)\right) G\left(M_{0}, M, s\right) d S(M) \\
& -\int_{S} \mu(M, s) \partial_{z(M)} G\left(M_{0}, M, s\right) d S(M)
\end{aligned}
$$

where $p\left(M_{0}, s\right)$ is the pressure at point $M_{0}, \rho_{f}$ is the fluid density, $s$ is Laplace parameter, $G\left(M_{0}, M, s\right)$ Green's elementary solution in an infinite medium, and $\mu(M, s)=$ $p_{+}(M, s)-p_{-}(M, s)$ a double layer potential, which expresses the pressure difference between the bottom and the top face of the plate at point $M$. The monopole part of the radiation is often neglected, since the normal velocity difference $u_{+}(M, s)-u_{-}(M, s)$ caused by the transverse strain along the thickness is found negligible in thin plates for bending modes. The dipole radiation term containing the diffraction effect is computed using the fluid-structure boundary condition $\partial_{z} p_{ \pm}(M, s)=-\rho_{f} s^{2} u_{ \pm}(M, s)$. This requires to evaluate the normal derivative of equation (7) when $M_{0}$ is located over the plate face:

$$
\begin{aligned}
\partial_{z\left(M_{0}\right)} p\left(M_{0}, s\right) & =\rho_{f} s^{2} \int_{S}\left(u_{+}(M, s)-u_{-}(M, s)\right) \partial_{z\left(M_{0}\right)} G\left(M_{0}, M, s\right) d S(M) \\
& -P . F \cdot \int_{S} \mu(M, s) \partial_{z\left(M_{0}\right)} \partial_{z(M)} G\left(M_{0}, M, s\right) d S(M)
\end{aligned}
$$

The second integral diverges and must be calculated as the finite part of Hadamard integral (P.F.). The first integral, which is weakly singular, is taken in the sense of Cauchy principal values (Filippi et al., 1999). It can be shown that

$$
\begin{aligned}
-\rho_{f} s^{2} u_{+}\left(M_{0}, s\right) & =\rho_{f} s^{2}\left(u_{-}\left(M_{0}, s\right)-u_{+}\left(M_{0}, s\right)\right) / 2 \\
& -P . F \cdot \int_{S} \mu(M, s) \partial_{z\left(M_{0}\right)} \partial_{z(M)} G\left(M_{0}, M, s\right) d S(M)
\end{aligned}
$$

Finally,

$$
\rho_{f} s^{2} \frac{u_{-}\left(M_{0}, s\right)+u_{+}\left(M_{0}, s\right)}{2}=P . F . \int_{S} \mu(M, s) \partial_{z}^{2} G\left(M_{0}, M, s\right) d S(M)
$$

This equation is a Fredholm integral equation of the first kind similar to classical results (Laulagnet, 1998) when the bottom and top face displacement is equal. The equation has 
been solved using a one-point collocation method. The boundary and finite element meshes coincide in the xy-plane so that a collocation point can be centered in each finite element. The displacement required by the boundary element program over each face is evaluated with the finite element solution and quadratic interpolation functions. In the idealized baffled configuration, based on the hypothesis of symmetry $u_{+}=-u_{-}$, the left hand side of equation (10) is equal to zero. The double layer potential $\mu(M, s)$ is therefore also equal to zero and no boundary element program is needed.

\subsubsection{The vibroacoustic problem}

Continuity of normal stresses reads

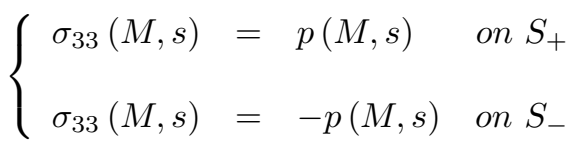

A vibroacoustic operator $\mathcal{P}(w, u, s)$ can be constructed by using the weak formulation of the classical finite element method. The product of the parietal pressure with a normal displacement $w$ is integrated over the plate faces:

$$
\mathcal{P}(w, u, s)=\int_{S_{+}} w\left(M_{0}\right) p\left(M_{0}, s\right) d S-\int_{S_{-}} w\left(M_{0}\right) p\left(M_{0}, s\right) d S
$$

When $M_{0}$ belongs to the top face, the indirect integral equation (7) is given by

$$
p\left(M_{0}, s\right)=-\mu\left(M_{0}, s\right) / 2
$$

$$
+\quad \rho_{f} s^{2} \int_{S}\left(u_{+}(M, s)-u_{-}(M, s)\right) G\left(M_{0}, M, s\right) d S(M)
$$

$\mu\left(M_{0}, s\right)$ changes of sign if $M_{0}$ belongs to the bottom face. Using (13), the vibroacoustic operator becomes

$$
\begin{aligned}
\mathcal{P}(w, u, s)= & \int_{S}-\left(w_{+}\left(M_{0}, s\right)+w_{-}\left(M_{0}, s\right)\right) \mu\left(M_{0}, s\right) / 2 \\
+ & \rho_{f} s^{2} \int_{S} \int_{S}\left(w_{+}\left(M_{0}, s\right)-w_{-}\left(M_{0}, s\right)\right) \\
& \times G\left(M_{0}, M, s\right)\left(u_{+}(M, s)-u_{-}(M, s)\right) d S(M) d S\left(M_{0}\right)
\end{aligned}
$$

The vibroacoustic system can finally be expressed in the following matrix form

$$
w^{T}\left[s^{2} M+K\right] u(s)-P^{h}(w, u, s)=w^{T}\left(F(s)+s I_{0}+V_{0}\right)
$$

where $P^{h}(w, u, s)$ is the discrete approximation of $\mathcal{P}(w, u, s) . \mathrm{K}$ and $\mathrm{M}$ are the structure stiffness and mass matrices, $u$ is displacement, $F(s)$ the frequency dependent excitation, $s I_{0}$ and $V_{0}$ the initial displacement and velocity, and $w$ is an arbitrary displacement vector.

\subsubsection{The perturbation method}

A weighting coupling parameter $\epsilon$ that varies from 0 in the uncoupled case to 1 in the fully coupled case is introduced. It is comparable, to some extent, with the small parameter introduced in the classical formalism of the light fluid approximation (Filippi et al., 2001), equal to the ratio between the fluid and solid density. The resonance value problem is written as

$$
w^{T}\left[s_{k}(\epsilon)^{2} M+K\right] u_{k}(\epsilon)=\epsilon P^{h}\left(w, u_{k}(\epsilon), s_{k}(\epsilon)\right),
$$


It consists in searching the resonance modes $u_{k}$ and their associated resonance values $s_{k}$. Deriving by $\epsilon$ yields

$$
w^{T}\left[s_{k}(\epsilon)^{2} M+K\right] \frac{\partial}{\partial \epsilon}\left(u_{k}(\epsilon)\right)+w^{T}\left[\frac{\partial}{\partial \epsilon}\left(s_{k}(\epsilon)^{2}\right) M\right] u_{k}(\epsilon)
$$

$$
=P^{h}\left(w, u_{k}(\epsilon), s_{k}(\epsilon)\right)+\epsilon \frac{\partial}{\partial \epsilon}\left(P^{h}\left(w, u_{k}(\epsilon), s_{k}(\epsilon)\right)\right)
$$

By setting $\epsilon=0, w=u_{k}(0)$ and reminding that $\left(s_{k}(0), u_{k}(0)\right)$ is the solution of the resonance value problem in vacuum, equation (17) becomes

$$
u_{k}^{T}(0)\left[\frac{\partial}{\partial \epsilon}\left(s_{k}(0)^{2}\right) M\right] u_{k}(0)=P^{h}\left(u_{k}(0), u_{k}(0), s_{k}(0)\right)
$$

And thus

$$
\frac{\partial s_{k}(0)}{\partial \epsilon}=P^{h}\left(u_{k}(0), u_{k}(0), s_{k}(0)\right) /\left[2 s_{k}(0) u_{k}^{T}(0) M u_{k}(0)\right]
$$

The first order approximation of the vibroacoustic resonance values is given by

$$
s_{k}(\epsilon)=s_{k}(0)+\epsilon \frac{\partial s_{k}(0)}{\partial \epsilon}
$$

In the fully coupled case $(\epsilon=1)$,

$$
s_{k}(1)=s_{k}(0)+P^{h}\left(u_{k}(0), u_{k}(0), s_{k}(0)\right) / 2 s_{k}(0)
$$

The acoustic radiation damping $\alpha$, expressed in $\mathrm{Hz}$, is finally deduced by computing the real part of $-s_{k}(1)$.

\subsubsection{Numerical results}

The acoustic radiation damping of the free aluminum plate studied in this paper has been computed. The plate main characteristics are summarized in table 2 . The numerical procedure has consisted of several steps. The finite element program introduced before and based on quadratic solid elements is used in a first step with a classical solver to compute the eigenpairs of the plate. In a second step, the complex scalar quantity $P^{h}$ is calculated from equation (14). This involves a boundary element program and integration routines that handle Green kernel singularity. A regularization is carried out by using a local element cylindrical coordinate system instead of the cartesian one. The integration is based on a Gauss-Legendre scheme. In a last step, frequencies in the air and acoustic damping are evaluated using equation (21). Numerical results of the 26 modes are displayed in figure 6 . It can be observed that acoustic damping is very low or even negligible for most of them. This behavior is expected since the coincidence frequency of the plate is close to $5970 \mathrm{~Hz}$ for the plate under consideration. However, radiation efficiency increases with frequency and some higher frequency modes experience damping by radiating.

\section{Material damping}

As pointed out by Cremer et al. (1988), two sources of dissipation mainly contribute to damping in aluminum. The first one, due to the thermoelastic behavior of the material, is quantified here numerically. The second one, due to friction micromechanisms that contribute to the viscoelastic behavior of the material, is then quantified by identification. 


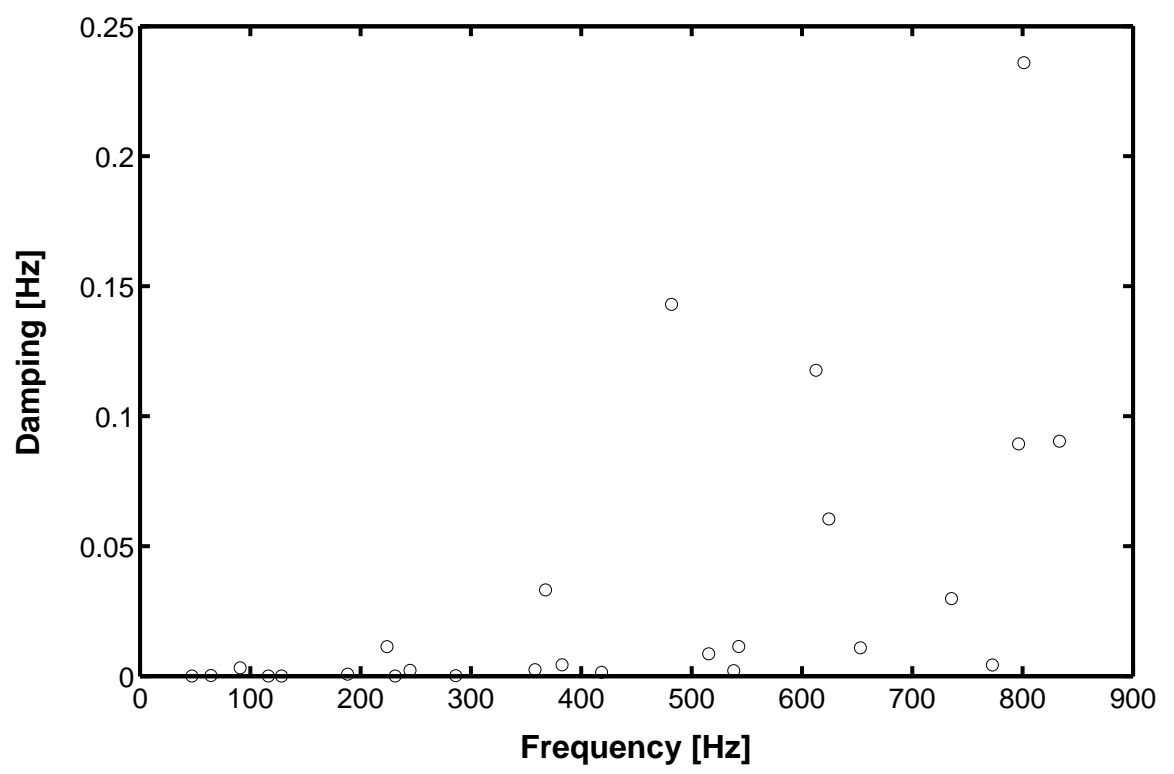

Figure 6: Computed acoustic damping of the $35 \mathrm{~cm} \times 40 \mathrm{~cm} \times 2 \mathrm{~mm}$ free aluminum plate

\subsection{Thermoelastic damping}

\subsubsection{Introduction}

Like acoustic radiation damping, thermoelastic damping is a coupling-related type of damping. It requires the analysis of an additional physical phenomenon, acoustic or thermal for instance, that alters the dynamic behavior of the structure. It also requires a new definition or an extension of the domain just as in vibroacoustics when the fluid domain is added to the solid domain. In the current thermoelastic case though, temperature and dynamic domains are identical since thermal effects occur within the plate. Thermoelastic damping is associated with an irreversible process during which heat flows by conduction from the hottest areas to the coldest ones. In line with the second thermodynamic law, entropy is created and vibratory energy is converted into heat. In the field of dynamics, isothermal elastic constants are usually used and no local temperature variation is observed. In acoustics, on the contrary, processes are regarded as adiabatic and a local temperature variation occurs but results in neither a heat transfer nor an irreversible process. Determining whether thermoelastic mechanisms can be regarded as adiabatic or isothermal is a difficult task; it is often somewhere in between, depending on the wave type and the geometry involved. The process can be considered as isothermal or relaxed when thermal relaxation occurs during a vibration period, while it is adiabatic if no equilibrium can be reached during this time. A key parameter governing the phenomenon is the distance between hot and cold regions. According to Lifshitz and Roukes (1999), this distance is related to the wavelength for longitudinal mechanical waves and to the thickness of the structure for flexural mechanical waves. Longitudinal waves are therefore associated with an adiabatic process in the lower frequency range and with an isothermal one in the upper frequency range, whereas the opposite is true for flexural waves. Shear waves are not coupled to the thermal field. 


\subsubsection{Equations}

After linearization, the classical thermoelasticity equations (CCT) of an isotropic material such as aluminum are given by the following expressions, in which Einstein summation convention is used (Nowacki, 1975):

$$
\left\{\begin{array}{lll}
\sigma_{i j} & =\delta_{i j} \lambda \epsilon_{k k}+2 \mu \epsilon_{i j}-\delta_{i j}(3 \lambda+2 \mu) \alpha\left(T-T_{A}\right) & \text { (elasticity eq.) } \\
k T_{, i i}=\rho c_{V} \dot{T}+\alpha T_{A}(3 \lambda+2 \mu) \epsilon_{\dot{k} k} & \text { (thermal eq.) }
\end{array}\right.
$$

where $\lambda, \mu$ are Lamé coefficients, $\mathrm{T}$ is the temperature, $\sigma_{i j}$ the stress tensor, $\epsilon_{i j}$ the strain tensor, $T_{A}$ the ambient temperature constant, $\rho$ the material density, $k$ the thermal conductivity, $c_{V}$ the specific heat per volume unit at constant strain, $\alpha$ the thermal expansion linear coefficient and $\delta_{i j}$ Kronecker symbol.

\subsubsection{Zener's thermoelastic model}

Zener (1948) has investigated dissipation in metals in great detail in the thirties. He has developed a model of thermoelastic damping for simply supported Euler-Bernoulli beams. Many authors are still using this model that can be considered as a standard reference in the field. It is based on the fundamental hypothesis that dissipation is mainly due to the first transverse thermal mode, which accounts for heat transfers within the beam thickness $\mathrm{h}$. The characteristic distance $\mathrm{d}$ between hot and cold parts is thus unique $(\mathrm{d}=\mathrm{h} / \pi)$ and associated with a unique relaxation time constant $\tau\left(\tau=d^{2} c_{V} / k\right)$. Zener's approximation therefore transforms the coupled thermoelastic system of equations into a unique equation of dynamics with a dissipation term. This one is characterized by Zener's rheological model, written as following:

$$
\left\{\begin{aligned}
Q^{-1} & =\Delta_{E} \frac{\omega \tau}{1+(\omega \tau)^{2}} \\
\Delta_{E} & =\frac{E_{a}-E_{i}}{E_{a} E_{i}}=\frac{E \alpha^{2} T_{A}}{c_{V}}
\end{aligned}\right.
$$

where $E_{a}$ is the adiabatic or unrelaxed modulus, $E_{i}$ the isothermal or relaxed modulus, and $c_{V}$ the heat capacity per unit volume. The value $E$ is the default isothermal value $\left(E=E_{i}\right)$. It is valid for flexural waves in the lower frequency range only. Zener's thermoelastic model is proportional: the computed loss factor applies to the whole strain field without any distinction, whereas the equations show that it should depend on the normal strain field. No modal dependence can therefore be expected in the computed damping values, only a smooth frequency one. More sophisticated analytical models have been developed since then, like the one by Li et al. (2012). According to this author though, it is not able to handle free boundary conditions properly as required here. An interesting discussion about four thermoelastic models of a thin plate with various degrees of approximation of the temperature field, in particular, can also be found in the paper by Norris (2006). The free boundary conditions case is not investigated directly yet, although some of the presented models may handle this specific boundary type.

\subsubsection{Numerical method}

As thermoelastic coupling is weak, it has been chosen to solve the thermomechanical problem by dealing with the thermal and dynamic equations separately, one after another. In order to gain some physical insights, the thermal problem has been investigated using a modal approach similar to the one used by Zener to model the temperature field. As almost no heat flows from the plate to the air due to its very low thermal conductivity, the temperature 
field in the plate can be expressed easily with a Fourier series. Thermoelastic coupling is handled using the following three steps procedure:

$1 \quad \begin{array}{r}\text { Solve the uncoupled dyn } \\ \sigma_{i j}=\delta_{i j} \lambda \epsilon_{k k}+2 \mu \epsilon_{i j}\end{array}$

Compute $\epsilon_{k k}$ in the form of a Fourier series (projection)

Solve the heat equation with a second member

2

$$
k T_{, i i}-\rho c_{V} \frac{\partial T}{\partial t}=\alpha T_{A}(3 \lambda+2 \mu) \frac{\partial \epsilon_{k k}}{\partial t}
$$

Deduce thermal stresses and build up a thermal stress matrix

$$
\sigma_{i j}^{t h}=-(3 \lambda+2 \mu) \alpha\left(T-T_{A}\right) \delta_{i j}
$$

3

Compute the thermoelastic strain energy $E_{t h}$

$$
E_{t h}=\int_{V} \epsilon_{i i} \sigma_{k k}^{t h}
$$

Deduce the thermomechanic system resonance values $\lambda_{t h}$

$$
\lambda_{t h}=\lambda+E_{t h} \quad \text { (after mass-orthonormalization, see eq. (6)) }
$$

In step 1, the dynamic problem is solved using classical tools such as the finite element program based on the solid 27-nodes element presented before and a real eigenvalue solver. Fourier coefficients are evaluated by projecting the computed normal strain on the Fourier basis. It is a time-consuming operation of numerical integration that requires a modal series truncation. A sufficient number of modes has been selected to observe a good convergence of the results. Once Fourier coefficients are known, the analytical solution of step 2 is straightforward to evaluate. The following non-homogeneous heat equation is solved in Laplace domain with homogeneous initial conditions:

$$
k T_{, i i}(s)-\rho c s T(s)=\alpha T_{A} s(3 \lambda+2 \mu) \epsilon_{k k}
$$

By positioning the plate normally to the z-axis so that all its coordinates are positive and a corner is located at point $(x=0, y=0, z=0)$, the zero heat flow boundary conditions result in a simple temperature field expression with cosines only:

$$
T(s)=\sum_{m, n, q}^{\infty} T_{m n q}(s) \cos (m \pi x / l) \cos (n \pi y / L) \cos (q \pi z / e)
$$

Each mode has its own frequency dependence, as required to be able to model non-proportionality correctly. A proportional damping such as the one described by Zener would have been modeled using a single frequency function for all modes. By inserting the temperature expression (25) into the homogeneous form of equation (24), thermal eigenvalues can be obtained:

$$
s_{m n q}=-\frac{k \pi^{2}}{\rho c}\left(\left(\frac{m}{l}\right)^{2}+\left(\frac{n}{L}\right)^{2}+\left(\frac{q}{e}\right)^{2}\right)
$$

A Fourier series of the normal strain can be written as following:

$$
\epsilon_{k k}=\sum_{m, n, q} A_{m n q} \cos (m \pi x / l) \cos (n \pi y / L) \cos (q \pi z / e)
$$

Numerical values of $\epsilon_{k k}$, obtained during step 1, are used to compute the coefficients $A_{m n q}$ 42 by projection. The coefficients of the temperature series can easily be deduced using

$$
T_{m n q}(s)=\frac{\alpha T_{A} s(3 \lambda+2 \mu) A_{m n q}}{\rho c\left(s_{m n q}-s\right)}
$$


${ }_{414} s_{m n q}$ has real values, while $\mathrm{s}$ is imaginary. This expression has thus no pole and no reso415 nance behavior can be observed in the temperature field. A thermal stress as well as an 416 associated finite element matrix are then computed. Each resonance value is finally updated 417 by projecting this matrix onto the mode subspace of the uncoupled system following the 418 perturbation approach described previously (equation (6)).

\subsubsection{Numerical results}

Numerical simulations of thermoelastic damping have been carried out for the considered plate as well as for a number of interesting configurations. The model main characteristics are summarized in table 3 . Figure 7 displays computed thermoelastic damping values of

\begin{tabular}{|l|l|}
\hline Plate dimensions $(\mathbf{x} \times \mathbf{y} \times \mathbf{z})$ & $35 \mathrm{~cm} \times 40 \mathrm{~cm} \times 2 \mathrm{~mm}$ \\
Mesh $(\mathbf{x} \times \mathbf{y} \times \mathbf{z})$ & $30 \times 30 \times 3$ \\
Number of thermal modes $(\mathbf{x} \times \mathbf{y} \times \mathbf{z})$ & $10 \times 10 \times 6$ \\
Thermal expansion linear coefficient $\alpha$ & $23.010^{-6} \mathrm{~K}^{-1}$ \\
Ambient temperature $T_{A}$ & $295.15 \mathrm{~K}$ \\
Heat capacity at constant pressure $C_{p}$ & $900.0 \mathrm{~J} /(\mathrm{K} . \mathrm{kg})$ \\
Thermal conductivity $k$ & $237.0 \mathrm{~W} /(\mathrm{m} . \mathrm{K})$ \\
\hline
\end{tabular}

Table 3: Thermomechanical properties of the aluminum plate model

422

423

about thirty modes in the clamped, simply supported and free boundary conditions cases. Zener's model is also represented. It is worth noting that Zener's damping model is accurate for clamped or simply supported boundary conditions but gives poor approximations of damping in the lower frequency range for free boundary conditions. The absence of link

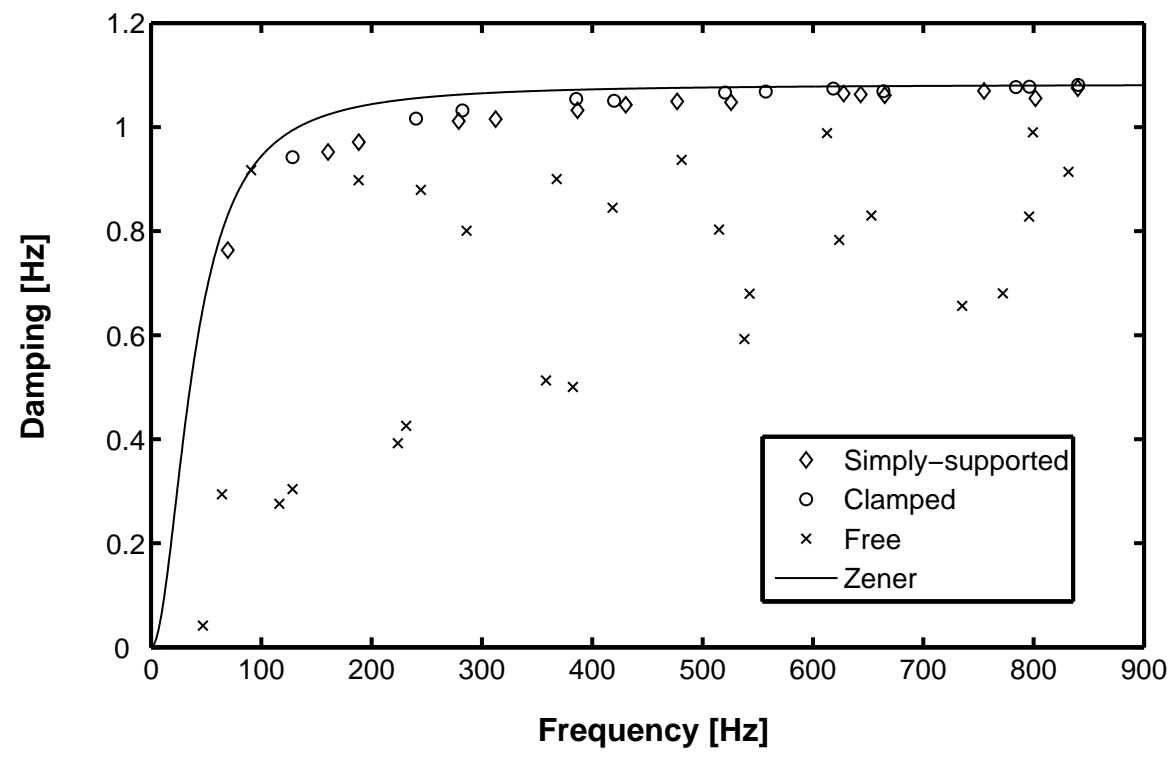

Figure 7: Comparison of the thermoelastic damping computed with various boundary conditions. Simply supported, clamped and free $35 \mathrm{~cm} \times 40 \mathrm{~cm} \times 2 \mathrm{~mm}$ aluminum plate

${ }_{427}$ between Zener's model of damping and the normal strain is noteworthy, since this last one is 
in fact directly associated to the local temperature variation. A better thermoelastic damping model inspired from the modal strain energy analysis could probably be implemented from the normal strain energy knowledge. Figure 8 illustrates this possibility in the free boundary conditions case and displays the ratio of the normal strain to the total energy as well as computed thermoelastic damping values. Simply-supported and clamped boundary conditions give an almost constant ratio of about $17 \%$. This is why a proportional damping model such as Zener's model can be applied in these cases.
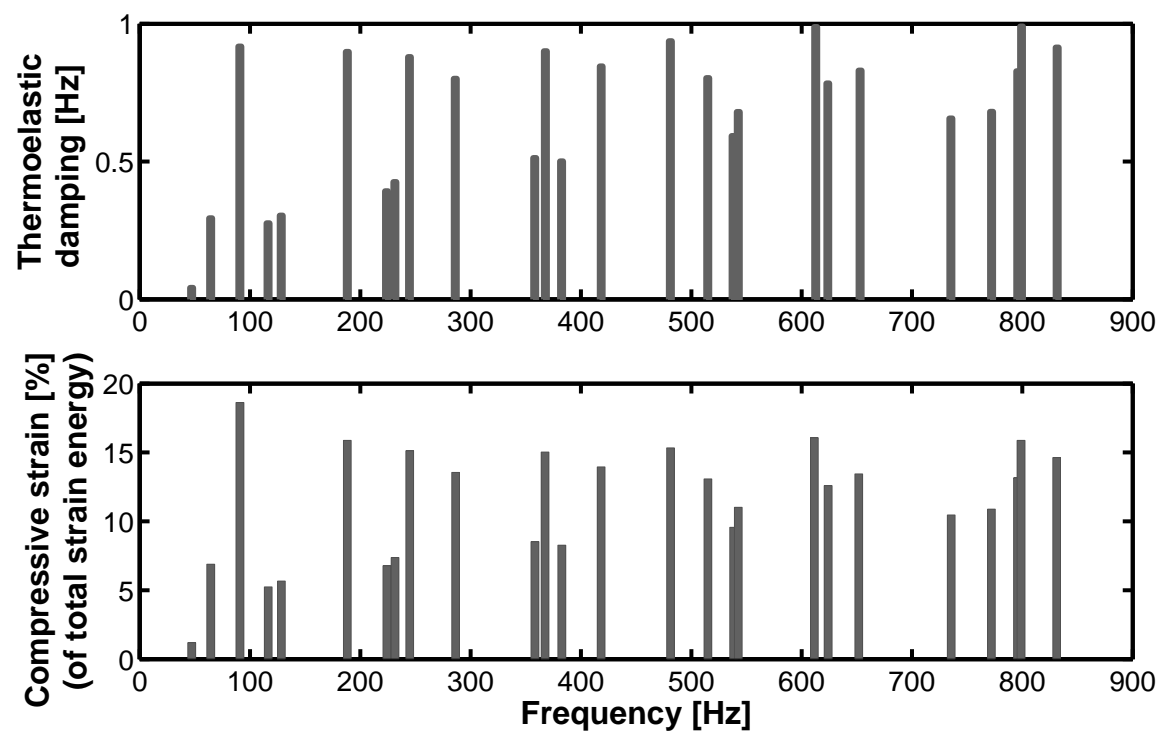

Figure 8: Comparison between the modeled thermoelastic damping and the ratio between the normal strain energy and the total strain energy. Free $35 \mathrm{~cm} \times 40 \mathrm{~cm} \times 2 \mathrm{~mm}$ aluminum plate

Figure 9 shows numerical estimations of the frequency shift due to thermoelastic coupling. The shift increases roughly linearly with respect to the frequency for all boundary conditions types. Thermoelastic coupling has thus a stiffening effect on the structure as pointed out also by Prabhakar et al. (2009) for the case of cantilever and doubly-clamped thermoelastic beams. Figure 10 and 11 present the contribution in percent of the most important thermal modes to the damping of the first structural modes. While figure 10 focuses on the first four modes of the free boundary conditions case, figure 11 compares the thermal modes contribution for various boundary conditions in the first structural mode case. The great majority of thermal modes have a component equal to one in the z-direction associated to a wavelength equal to twice the thickness. This confirms Zener's prediction that the first thermal transverse mode is responsible for most of the thermoelastic dissipation. It is also worth noting that each structural mode is associated with a very specific combination of thermal modes that strongly varies depending on the boundary conditions. Figure 12 finally gives an illustration of the influence of the thickness $h$. Zener pointed out that thermoelastic damping has a $1 / h^{2}$ dependence by carrying out a limit calculation. The approximation is only valid in the upper frequency range above the characteristic frequency $f_{c}\left(f_{c}=\pi^{2} k / h^{2} c_{V}\right)$. The numerical results confirm the approximation quality, since a damping value of about $0.2,0.8$ and roughly 3.5 is obtained for $4-\mathrm{mm}, 2-\mathrm{mm}$ and $1-\mathrm{mm}$ thick plates, respectively. 


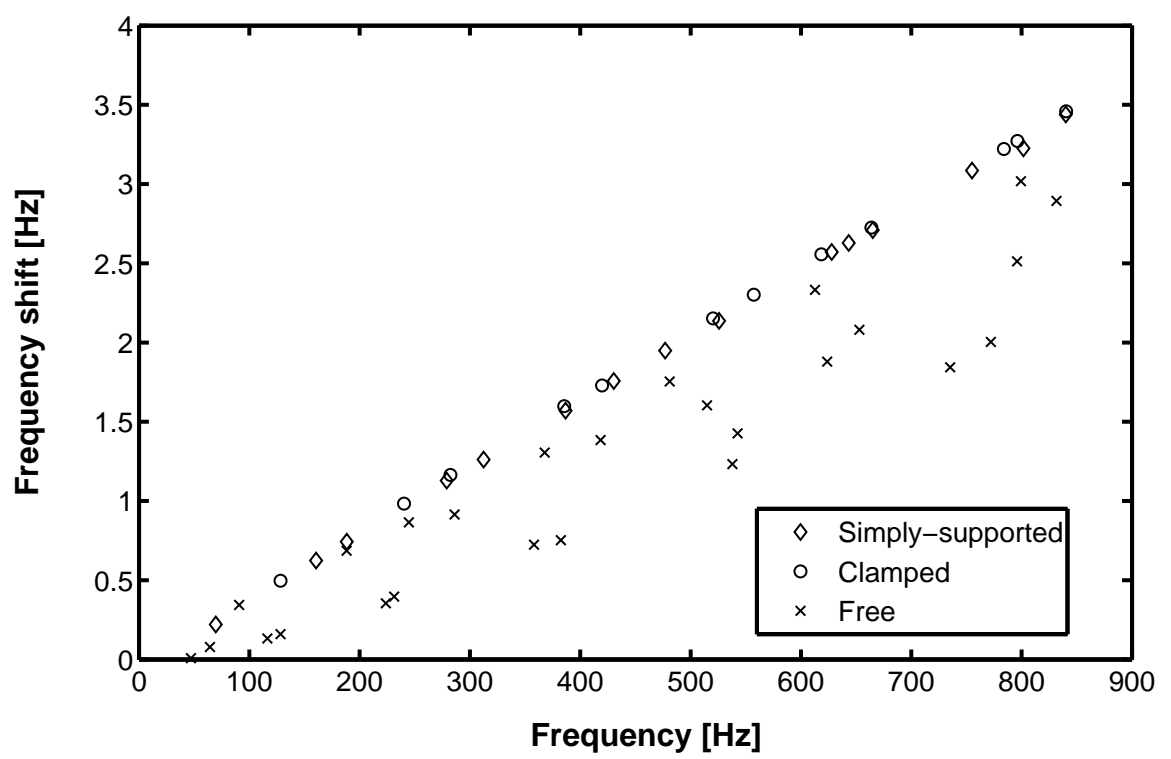

Figure 9: Comparison of the frequency shift due to the thermoelastic coupling for various boundary conditions. Simply supported, clamped and free $35 \mathrm{~cm} \times 40 \mathrm{~cm} \times 2 \mathrm{~mm}$ aluminum plate

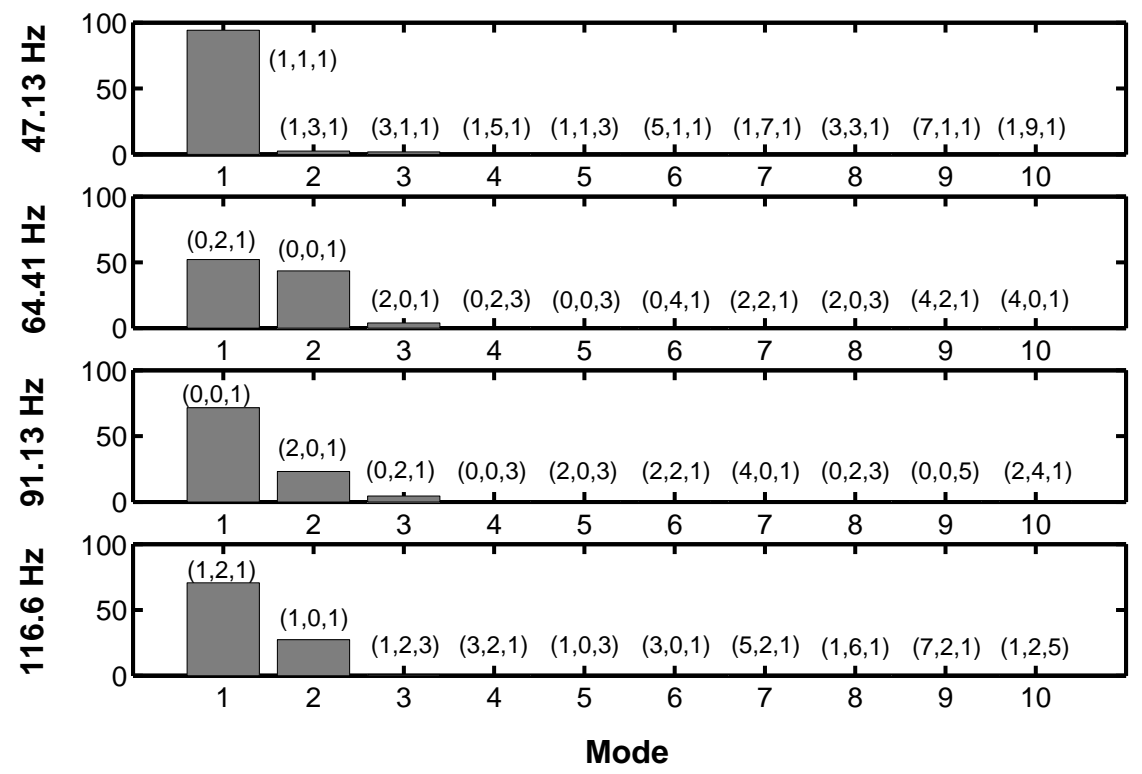

Figure 10: Thermal modes that most contribute most to the thermoelastic damping of a free $35 \mathrm{~cm} \times 40 \mathrm{~cm} \times 2 \mathrm{~mm}$ aluminum plate. Contributions as a percent of total damping 

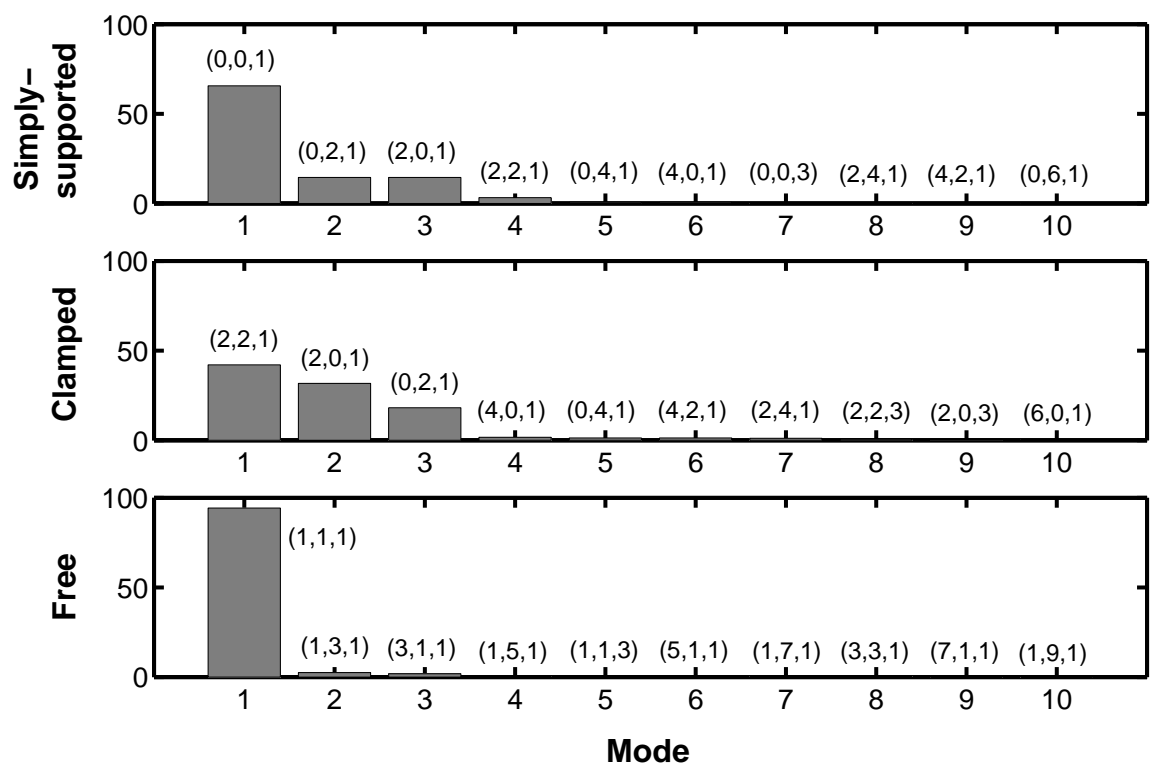

Figure 11: Thermal modes that most contribute to the first mode thermoelastic damping. $35 \mathrm{~cm} \times 40 \mathrm{~cm} \times 2 \mathrm{~mm}$ aluminum plate with free, simply-supported and clamped boundary conditions. Contributions expressed as a percent of total damping

454

\subsection{Viscoelastic damping}

The remaining main damping component of an aluminum plate is referred to as viscoelastic damping. It is is due to a local viscoelastic dissipation process caused by crystallographic defects or irregularities such as dislocations, interstices or grain boundaries, that essentially depend on the material and the manufacturing process. Despite the existence of fine micromechanical models (Granato-Lücke model (1956)), it is still impossible to quantify this component correctly without making any measurement. Many references can provide with damping values identified experimentally in the literature. However, most of them focus on the effects of temperature on the aluminum in the very low frequency range rather than on the frequency itself, probably because measurements in higher frequency ranges are particularly difficult to carry out. Rivière (2004) has obtained a damping of $Q^{-1} \sim 0.003$ at $0.01 \mathrm{~Hz}$ in a polycrystalline aluminum at room temperature. Wei et al. (2002) have measured internal friction values of about $Q^{-1} \sim 0.001$ in aluminum at $1 \mathrm{~Hz}$, and Wang et al. (2000) a value of $Q^{-1} \sim 0.0036$ at room temperature. In a broader frequency range, Cremer et al. (1988) have reported a constant frequency value of $Q^{-1} \sim 0.0001$ at room temperature.

In order to analyze the remaining part of damping in aluminum, a direct comparison between computed damping (thermoelastic and acoustic damping) and the measured one is displayed in figure 13. Since all other main dissipation sources (air flow and attachment dissipation) have been found or are assumed negligible, the difference between these quantities is supposed to be a good approximation of viscoelastic damping. It is confirmed, at least partially, by observing that the difference matches a straight line. If another damping unit such as the loss factor or the inverse quality factor is used, the damping is found to 


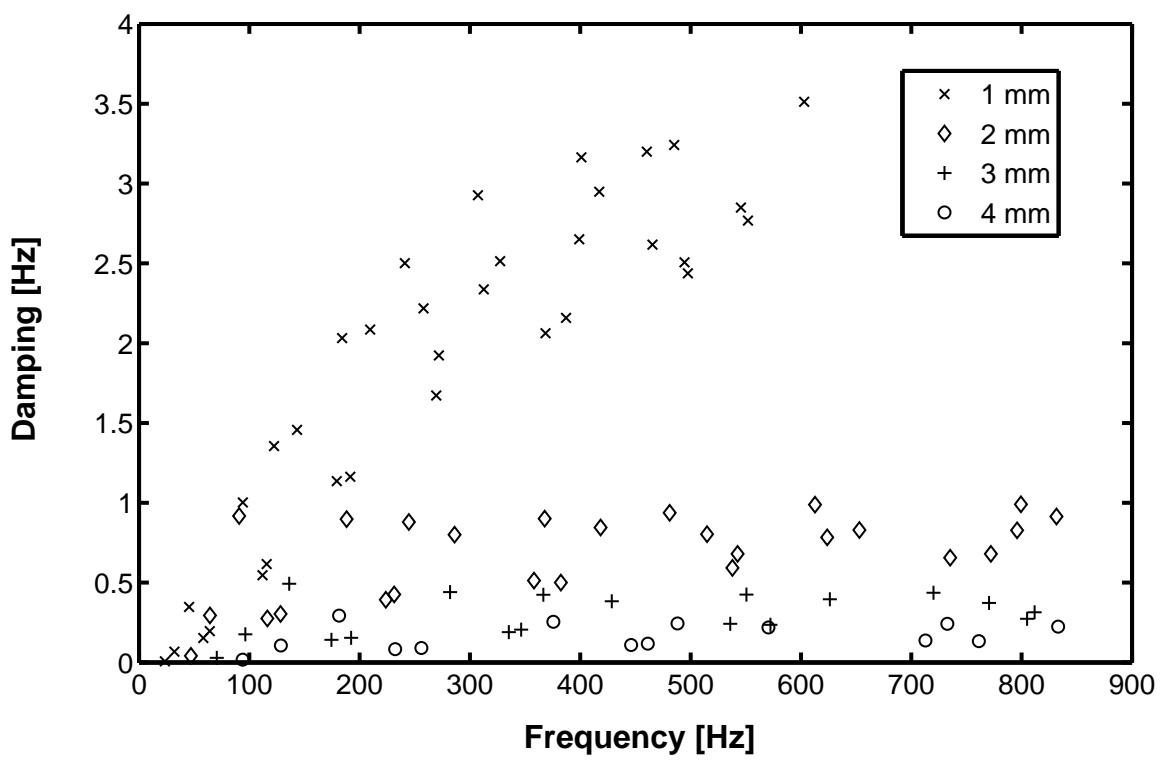

Figure 12: Thermoelastic damping computed for various plate thicknesses. First 35 modes in a free $35 \mathrm{~cm} \times 40 \mathrm{~cm} \times 1-2-3-4 \mathrm{~mm}$ aluminum plate

be a constant function of frequency equal to $Q^{-1}=0.00037$. This value is quite consistent with the one given by Cremer et al. (1988), but it is below other values mentioned in the literature.

\section{Conclusions}

A detailed analysis of the dissipation sources acting in a thin suspended aluminum plate in the low frequency range $(0-900 \mathrm{~Hz})$ has been carried out in this paper. Air viscosity and noise radiation have been modeled to account for the fluid influence on damping. Air viscosity has been considered by combining Landau and Lifschitz stationary flow analysis to a finite element capability, while acoustic radiation has been simulated with the same capability and a boundary element program. A perturbation technique has finally been implemented to observe how modes frequency and damping are shifted by both phenomena. While the effect of air viscosity on the plate overall damping has been found negligible, the low acoustic damping values increase with frequency and become substantial for a couple of modes in the higher part of the considered frequency domain. Thermoelastic damping has been computed by using analytical Fourier series of the temperature field, the same finite element program and perturbation technique. Aluminum viscoelastic damping has been identified by subtracting the computed values of the thermoelastic and acoustic damping to the one measured with a contact-free modal analysis and the logarithmic decay method.

It is found almost constant over a broad range of frequencies when damping is expressed as a loss factor or as the inverse of a quality factor. The general methodology proposed in this paper, which consists in a systematic analysis of damping sources, thus provides an efficient means of gaining insight into the dynamics of systems with very low damping such as aluminum structures.

Acknowledgments 


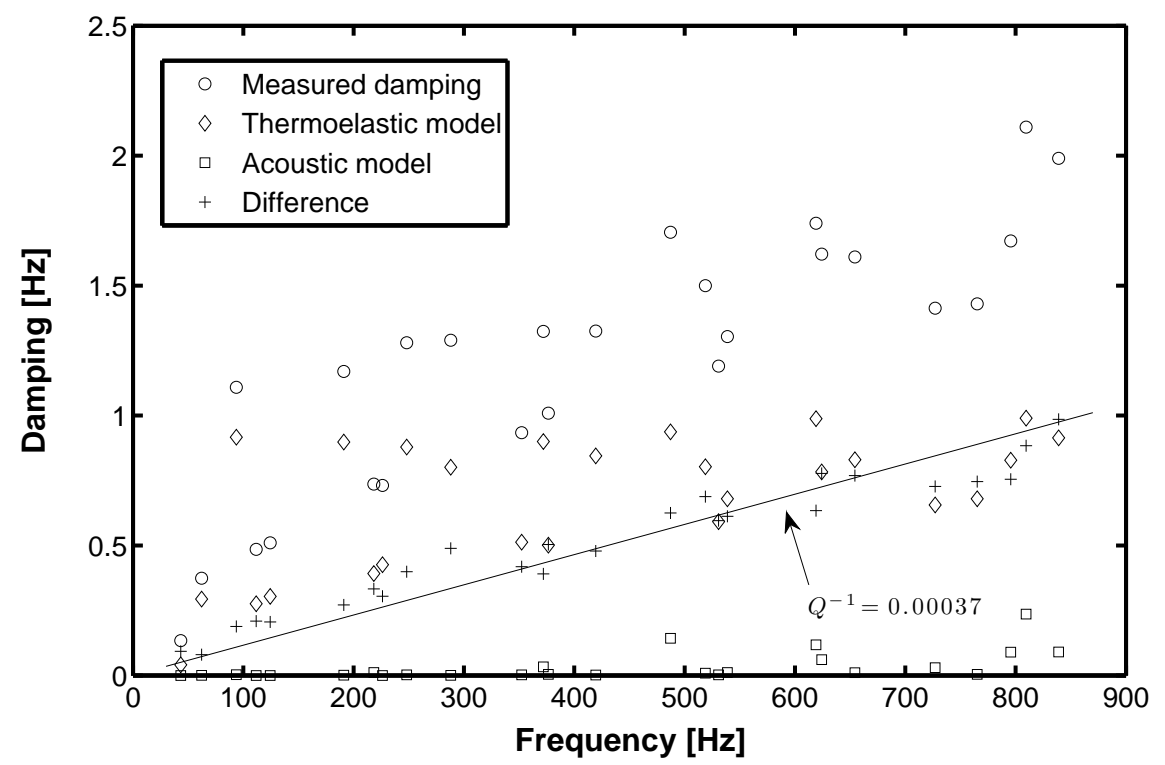

Figure 13: Experimental/modeled thermoelastic damping. Free $35 \mathrm{~cm} \times 40 \mathrm{~cm} \times 2 \mathrm{~mm}$ aluminum plate

This research received no specific grant from any funding agency in the public, commercial, or not-for-profit sectors.

\section{References}

[1] Adhikari S (2011) An iterative approach for non proportionally damped systems. Mechanics Research Communications 38(3): 226-230.

[2] Atalla N, Nicolas J, Gauthier C (1996) Acoustic radiation of an unbaffled vibrating plate with general elastic boundary conditions. Journal of the Acoustical Society of America 99(3): 1484-1494.

[3] Cha PD (2005) Approximate eigensolutions for arbitrarily damped nearly proportional systems. Journal of Sound and Vibration 288(4-5): 813-827.

[4] Cortés F, Elejabarrieta MJ (2006) An approximate numerical method for the complex eigenproblem in systems characterised by a structural damping matrix. Journal of Sound and Vibration, 296(1-2): 166-182 .

[5] Côté AF, Atalla N and Guyader JL (1998) Vibroacoustic analysis of an unbaffled rotating disk. Journal of the Acoustical Society of America 103(3): 1483-1492.

[6] Cousteix J (1988) Couche limite laminaire. Toulouse: Cépaduès.

[7] Cremer L, Heckl M and Ungar EE (1988) Structure-Borne Sund. Berlin: Springer.

[8] Cuesta C and Valette C (1993) Mécanique de la corde vibrante. Paris: Hermès.

[9] Filippi PJT, Habault D, Lefebvre J-P, Bergassoli A (1999) Acoustics. San Diego: Academic Press. 
[10] Filippi PJT, Habault D, Mattei PO, Maury C (2001) The role of the resonance modes in the response of a fluid-loaded structure. Journal of Sound and Vibration 239(4): 639-663.

[11] Granato AV and Lücke K (1956) Theory of mechanical damping due to dislocations. Journal of Applied Physics 27(6): 583-593.

[12] Lambourg C and Chaigne A (2001) Time-domain simulation of damped impacted plates. Journal of the Acoustical Society of America 109(4): 1422-1432.

[13] Landau LD and Lifschitz EM (1986) Fluid Mechanics. Oxford: Pergamon Press.

[14] Laulagnet B (1998) Sound radiation by a simply supported unbaffled plate. Journal of the Acoustical Society of America 103(5): 2451-2462.

[15] Li P, Fang Y and Hu R (2012) Thermoelastic damping in rectangular and circular microplate resonators. Journal of Sound and Vibration 331(3): 721-733.

[16] Lifshitz R and Roukes ML (1999) Thermoelastic damping in micro- and nanomechanical systems. Physical Review B 61(8): 5600-5609.

[17] Nashif AD, Jones DI and Henderson JP (1985) Vibration Damping. New-York: John Wiley \& sons.

[18] Norris AN (2006) Dynamics of thermoelastic thin plates: A comparison of four theories. Journal of Thermal Stresses 29(2): 169-195.

[19] Nowacki W (1975) Dynamical Problems of Thermoelasticity. Warszawa: Polish Scientific Publishers.

[20] Prabhakar S, Païdoussis MP and Vengallatore S (2009) Analysis of frequency shifts due to thermoelastic coupling in flexural-mode micromechanical and nanomechanical resonators. Journal of Sound and Vibration 323(1-2): 385-396.

[21] Rivière A (2004) Analysis of the low frequency damping observed at medium and high temperatures. Materials Science and Engineering A 370(1-2): 204-208.

[22] S.S. Rao (2010) Mechanical Vibrations, Fifth Edition. Upper Saddle River: Prentice Hall.

[23] Wang J, Zhang Z and Yang G (2000) The dependence of damping capacity of PMMCs on strain amplitude. Computational Materials Science 18(2): 205-211

[24] Wei JN, Gong CL, Cheng HF, Zhou ZC, Li ZB, Shui JP and Han FS (2002) Lowfrequency damping behavior of foamed commercially pure aluminum. Materials Science and Engineering A 332(1-2): 375-381

[25] Woodhouse J (1988) Linear damping models for structural vibration. Journal of Sound and Vibration 215(3): 547-569.

[26] Zener C (1948) Elasticity and Anelasticity of Metals. Chicago: The University of Chicago Press. 